\title{
Anesthetic experience for laparoscopic cholecystectomy in a patient with myelodysplastic syndrome with trisomy 8 mosaicism syndrome
}

\author{
Mae Hwa Kang, Kyeung Sin Sim, Yi Hwa Choi, Soo Kyung Lee, and Eun Young Park \\ Department of Anesthesiology and Pain Medicine, Hallym University Sacred Heart Hospital, Anyang, Korea
}

The trisomy 8 mosaicism syndrome (T8M) is a chromosomal disorder due to the presence of a complete extra chromosome 8 in some cells of the body. The anesthetic management of the patient may provide some problems such as a difficult tracheal intubation, cardiac malformation, hematological malignancies, mental retardation and kidney dysfunction $[1,2]$. This seems to be the first report on myelodysplastic syndrome patients with T8M who experienced a laparoscopic cholecystectomy under general anesthesia.

A 58-year-old male patient $(173 \mathrm{~cm}$ height and weighed 53 $\mathrm{kg}$ ) was scheduled for preventive laparoscopic cholecystectomy due to gallstones. Recently, he was diagnosed with myelodysplastic syndrome with T8M and it was decided to perform the operation before the beginning of chemotherapy. He had undergone a percutaneous coronary intervention with drug eluting stent due to stable angina 3 months ago and had been taking dual antiplatelet therapy (aspirin and clopidogrel). The preoperative routine laboratory values showed bicytopenia, PT/aPTT prolongation and an elevation of liver enzymes. The stability of the cervical spine was reviewed and showed a degenerative spondylosis. The findings of electrocardiography and echocardiography were normal. The patient exhibited the normal facial features. On airway examination, the thyromental distance showed $4.5 \mathrm{~cm}$, the Mallampati airway classification was grade 3 and difficulties with an intubation were predicted. The blood pressure was $110 / 70 \mathrm{mmHg}$, heart rate was 70 beats/min and oxygen saturation was $98 \%$ before anesthesia. The patient was premedicated with intravenous glycopyrrolate $0.1 \mathrm{mg}$ before the induction of anesthesia. Intraoperative monitoring was performed including pulse oximetry, electrocardiogram, capnogram, bispectral index and direct arterial pressure monitoring via radial arterial cannula. After preoxygenation, anesthesia was induced with $200 \mathrm{mg}$ of thiopental and $0.5 \mu \mathrm{g} / \mathrm{min} / \mathrm{kg}$ of remifentanil. After confirming the adequate mask ventilation, $40 \mathrm{mg}$ of rocuronium was given to facilitate the endotracheal intubation. As expected, the endotracheal intubation was not easy. Lightwand and Airtraq ${ }^{\circledR}$ were prepared because of the predicted difficulties in intubation. It failed using a Macintosh laryngoscope and $7.5 \mathrm{~mm}$ endotracheal tube using stylet and Sellick's maneuver. We successfully intubated using Airtraq ${ }^{\circledR}$ after we confirmed a Cormack and Lehane airway classification of Grade 3. The mechanical ventilation was started with volume controlled ventilation with a tidal volume of $550 \mathrm{ml}$ and a respiratory rate of $13 / \mathrm{min}$. The anesthesia was maintained with 50\% oxygen/air, 1.5-2 vol\% of sevoflurane and remifentanil at $0.3 \mu \mathrm{g} / \mathrm{min} / \mathrm{kg}$. At the end of surgery, fentanyl 40 $\mu \mathrm{g}$ was used to control pain, $0.2 \mathrm{mg}$ of glycopyrrolate and $1 \mathrm{mg}$ of neostigmine were injected to reverse a muscle paralysis. The patient was extubated and transferred to the intensive care unit with $100 \%$ oxygen supplemented by a face mask.

Trisomy 8 is a rare chromosome disorder characterized by three copies of chromosome 8 in some cells of the body. A complete trisomy 8 affects the fetal development and results in an abortion or early lethal condition. T8M is a condition that occurs when only a portion of these cells includes three copies

Corresponding author: Eun Young Park, M.D., Ph.D., Department of Anesthesiology and Pain Medicine, Hallym University Sacred Heart Hospital, 22, Gwanpyeong-ro, 170 beon-gil, Dongan-gu, Anyang 431-796, Korea. Tel: 82-31-380-3953, Fax: 82-31-385-3244, E-mail: parkey00@hanmail.net (c) This is an open-access article distributed under the terms of the Creative Commons Attribution Non-Commercial License (http:// creativecommons.org/licenses/by-nc/3.0/), which permits unrestricted non-commercial use, distribution, and reproduction in any medium, provided the original work is properly cited. 
of chromosome 8 , while other cells contain the usual two copies of chromosome 8 . T8M is usually characterized by distinct facial features, including palate abnormalities, eye abnormalities, a bulbous tipped nose, low-set or abnormally shaped ears, bone and tissue abnormalities, various structural heart problems, hydronephrosis, cryptorchidism, mental delay and deep hand and feet creases. These characteristics are manifested by various appearances [1].

T8M as the only abnormality is the most common karyotype finding in acute myeloid leukemia and myelodysplastic syndromes (MDS), occurring in approximately 5 and $10 \%$ of the cytogenetically abnormal cases [2].

The concern associated with the anesthetic management in a patient with $\mathrm{T} 8 \mathrm{M}$ is a potential difficult intubation because of a high arched palate, cleft palate and micrognathia [1]. In our case, there were no cleft problems, but the airway management was anticipated to be difficult according to the thyromental distance and Mallampati airway classification. Thereafter, the intubation using an Airtraq ${ }^{\circledR}$ was successful. Some of the common musculoskeletal features of T8M include skull malformations such as macrocephaly, frontal bossing, shape malformations and vertebral number and/or spine deformities such as scoliosis or kyphosis, symmetrical widening of the clavicles, a long and narrow thorax with wide sloping ribs, a peculiar shape of the pelvis with an increased iliac angle and coxa valga, hand and foot anomalies including clinodactyly, radial deviation of fingers with or without contractures and sternum anomalies (usually pectus excavatum) [3]. We have to evaluate those musculoskeletal anomalies during the pre-anesthetic visit because these may affect the tracheal intubation, mechanical ventilation and the intraoperative positioning.

A preoperative evaluation of the cardiac function is essential, especially because there is the possibility of congenital cardiac malformations such as pulmonary stenosis, holes in the heart wall and persistent ductus arteriosus [1]. In our case, echocardiography was done to evaluate cardiac malformations and confirm normal cardiac functions in spite of history of a stable angina. The frequency of malformations of the urinary system is much higher in patients with chromosome abnormalities than in the general population [1]. So, the kidney function should also be evaluated preoperatively. The degree of mental retardation varies from mild to moderate [1]. Some T8M patients may have seizures, so, their history of seizures and medication has to be checked preoperatively also.

The most commonly mentioned eye problems in the T8M are strabismus and corneal clouding. Anwar et al. [4] reported very different ophthalmic features like bilateral Duane's syndrome, congenital pendular nystagmus, bilateral myopic astigmatism and macula hypoplasia with or without significant vision problems. During the operation, we should pay attention to the eye care. MDS patients with a cytogenic abnormality of T8M also had Behçet's disease-like symptoms [5].

In conclusion, T8M is a rare chromosomal abnormality with a multisystem disorder. It may be associated with difficult intubation, hematological malignancies, facial deformities, musculoskeletal anomalies, cardiac malformation, kidney dysfunction, mental retardations, eyes and skin problems. Because patients with hematologic malignancies may be associated with T8M, the pre-anesthetic evaluation of the patient should be thoroughly performed to find the appropriate anesthesia.

\section{References}

1. Kosztolànyi G, Bühler EM, Elmiger P, Stalder GR. Trisomy 8 mosaicism. A case report and a proposed list of the clinical features. Eur J Pediatr 1976; 123: 293-300.

2. Paulsson K, Johansson B. Trisomy 8 as the sole chromosomal aberration in acute myeloid leukemia and myelodysplastic syndromes. Pathol Biol (Paris) 2007; 55: 37-48.

3. Silengo MC, Davi GF, Franceschini P. Radiological features in trisomy 8. Pediatr Radiol 1979; 8: 116-8.

4. Anwar S, Bradshaw K, Vivian AJ. Ophthalmic manifestations of trisomy 8 mosaic syndrome. Ophthalmic Genet 1998; 19: 81-6.

5. Kimura S, Kuroda J, Akaogi T, Hayashi H, Kobayashi Y, Kondo M. Trisomy 8 involved in myelodysplastic syndromes as a risk factor for intestinal ulcers and thrombosis--Behçet's syndrome. Leuk Lymphoma 2001; 42: 115-21. 